\title{
On the electronic properties of some metals using density-functional theory
}

\author{
Mustapha Lateef O. ${ }^{1 *}$, Oyewande Oluwole E. ${ }^{2}$, Kolebaje Olusola T. ${ }^{3}$ \\ ${ }^{I}$ Department of Physics, Lagos State Polytechnic, Ikorodu, Nigeria \\ ${ }^{2}$ Department of Physics, University of Ibadan, Ibadan, Nigeria \\ ${ }^{3}$ Department of Physics, Adeyemi College of Education, Ondo, Nigeria \\ *Corresponding author E-mail: muslaty2k@gmail.com
}

\begin{abstract}
The use of density functional theory as a viable tool for investigating the electronic structure of both periodic and disordered system at ground state has been emphasized. In this study, we employ local density approximation and plane wave formalism via Quantum ESPRESSO, the selected metals was observed to exhibit degenerate but overlap bands except Copper and Silver whose inner d state function transits its broader s state. The study shows the efficacy of density functional theory as a promising tool for explaining electronic properties of metal.
\end{abstract}

Keywords: Density Functional Theory, Electronic properties, Local Density Approximation, Quantum ESPRESSO

\section{Introduction}

Solving the total energy of a given system at its ground-state, is a highly sought after quantity. Many, if not most, physical properties of solids (or materials) can be related to total energies. Density-Functional Theory (DFT) and many other methods have been designed to solve this problem. The pros and cons of the various methods are discussed in depth in literature depending on the choice formalism to describe the system. This study is motivated by veracity of the method which is based DFT/LDA scheme, physical clarity and relatively modest computational task required for this study.

Electronic properties distinguish material due behavior of their electrons; particularly the existence of prohibited regions of energy and the magnitude in their electronic excitation spectral. However, the study of the electronic structure of material requires the approximation of the eigenvalue problem or total energy of complex many-body problem describing such system. Owing to the development of Density Functional Theory (DFT) in the mid 1960's [12], density functional theory is a quantum mechanical modelling tool used in computational solid state physics to investigate the electronic structure, magnetic properties, stability (phonon dispersion) and mechanical behavior (tensile strength, fracture and plasticity) [3] of large molecules as well as systems made of many hundreds of atoms at ground state. The advantage of the theory is that the ground state energy is obtained without involving individuals of electron wave function. Density-functional theory generously demonstrated that the electron density of a fully interacting system could actually, in a rigorous way, be obtained from simple one-electron theory [4], except for the strongly influenced inherent interaction between particles obtained by statistical approximation called exchange and correlation functionals.

Electronic properties obtained by DFT-based calculations are in general comparable with experimental values, as long as the relevant computational parameters such as the choice of basis functions and the representation of electrons correlation are properly chosen [3]. In the beginning of seventies till recent years Journals were full of LDA calculations among several other intricate methods of approximating the exchange and correlation function where the agreement with experiment was almost perfect and remaining discrepancies were blamed on numerical flaws. The systematic differences between electronic structures calculated with local density approximation and measurement made with angle-resolved photoelectron spectroscopy (ARPES) even for metals [5] resolved the consequence of the fact that the experimental measurements probe the excitation spectral of the solid while local density approximation only gives the ground state properties $[4,6]$. 
This study aims to employ the sophisticated technique of density functional theory via local density approximation in light of ab initio commercial software package called Quantum-ESPRESSO (opEn Source Package for Research in Electronic Structure, Simulation and Optimization).

\section{DFT description}

For a set of fixed nuclear position, density-functional theory expresses the ground state energy of many-electron system as a unique functional of the electronic density of the form.

$E[n(r)]=T[n(r)]+V_{C}[n(r)]+E_{x c}[n(r)]$

Where $\mathrm{T}$ and $\mathrm{V}_{\mathrm{c}}$ are known functionals corresponding to kinetic energy of electrons and the potential energy of electronnucleus (or electron-ion) and nucleus-nucleus (or ion-ion) Coulomb interactions, in that order. Eqn. (1) represents an enormous simplification of the many-electron problem. Unfortunately, the exact functional dependence of $E n(r)$ was not known till Kohn and Sham (2) proposed a practical scheme by mapping the problem of $\mathrm{N}$ interacting electrons in an external potential onto a fictitious set of $\mathrm{N}$ non interacting electrons in an effective potential and as the electron-ion potential provided by the fixed nuclei. Using this scheme, equation (1) the Hamiltonian can be expressed as

$E[n(r)]=T_{0}(n(r))+\int V_{e x t} n(r) d^{3} r+\frac{e^{2}}{2} \iint \frac{n(r) n\left(r^{\prime}\right)}{\left|r-r^{\prime}\right|} d^{3} r d^{3} r^{\prime}+E_{x c}[n(r)]$

where $T_{0}$ is the kinetic energy of auxiliary non-interacting system with the same density, the second and third terms on the right are the Hartree energy and electron-ion interaction energy respectively, and $E_{x c}[n(r)]$ is the remaining exchange-correlation energy. Equation (2) can be shown to lead to a set of one-particle Schrödinger-like equations:

$\left[\frac{\hbar^{2}}{2 m} \nabla^{2}+V_{e f f}(r)\right] \psi_{i}(r)=E_{i} \psi_{i}(r)$

$E_{i}$ is the Kohn-Sham eigenvalues, $\psi_{i}(r)$ is the Kohn-Sham single particle orbitals and ground state density spatial function given by,

$\mathrm{n}(r)=\sum_{\text {no.of occ }}\left|\psi_{i}(r)\right|^{2}$

Summing over occupied state, and an effective potential is expressed by

$V_{e f f}(r)=V_{e x t}+e^{2} \int \frac{n\left(r^{\prime}\right)}{\left|r-r^{\prime}\right|} d^{3} r^{\prime}+\frac{d\left(E_{x c}\right)}{d n}$

The first term tells about the electron-ion interaction, second term represents the Hartree potential, i.e., the classical electrostatic interaction and the last term is the exchange-correlation potential $E_{x c}$ and can be obtained by

$$
E_{x c}=\int n(r) \varepsilon_{x c}[n(r)] d r
$$

Approximation for the exchange and correlation potential, $E_{x c}$ can be carried out by, LSDA (Local Spin Density Approximation), GGA (Generalized Gradient Approximation), MGGA (Meta generalized gradient approximation) and LDA (Local Density Approximation). LDA is the exchange-correlation energy per particle of the homogeneous electron gas of density $n$, i.e., the exchange-correlation energy density is taken to be that of a uniform electron gas of the same density, specified by

$$
E_{x c}{ }^{L D A}[n]=\int d^{3} r n(r) \varepsilon_{x c}{ }^{H E G} n(r)
$$

$E_{x c}$ is the exchange-correlation energy per electron in a homogeneous electron gas (HEG) of constant density (7). The Kohn-Sham equation [2] given in equation (2) is solved for a set of fixed nuclear (ionic) positions by initial assumption of $\psi_{1}(\mathrm{r})$, obtained from a set of exact basis functions. The plane waves that give the solution to the Schrödinger equation for a free electron, while the participation coefficients are optimized in a way similar to the Hartree-Fock method, set of 
improved Kohn-Sham wavefunction $\psi_{\mathrm{i}}(\mathrm{r})$ is next used to compute a more accurate density function, according to equation (4). This iterations repeat until the exchange-correlation energy and the density converge to within some tolerance.

\section{Computational approach}

Total electronic energy of the elemental Sodium, Potassium, Copper and Silver have been calculated using Self Consistent Field method and its electronic structure presented in momentum space within the LDA as embedded in Quantum ESPRESSO. We employ plane wave self consistent field (PWSCF) simulation code to perform the total energy calculations by input of appropriate parameter that describe the features of the selected metals i.e. atom dimension, braivais type, choice pseudopotential, lattice constant, etc and electronic exchange and correlation specified by Perdew-Zunger (PZ) [8]. The cell dimension, energy cut-offs and the k-points used in the study were obtained from their respective convergence test. The large plane wave energy cut off presented for copper and silver is attributed to the highly localized d electrons and the electronic energy were plotted against high symmetry k points of these metals using monk horst-park grid [9].

\section{Results and discussion}

Electronic structure of these metals ( $\mathrm{Na}, \mathrm{K}, \mathrm{Cu}$ and $\mathrm{Ag}$ ) has been approximated using Quantum ESPRESSO 4.2.1 and result presented in the figures below. The plane waves represent the various electron state of the metal, and dashed line lying along the $0 \mathrm{eV}$ is the Fermi level, separating the maximum occupied state from the unoccupied state. The labels along the horizontal and vertical axis correspond to k-space points in the Brillouin zone [10] and the corresponding energy respectively.

Figures $1 \& 2$ are the electronic structure of sodium and potassium within the local density approximation of density functional theory respectively, shows quite similar but degenerate or overlap of states along the high symmetry lines but was intense in lower state of potassium, Figures $3 \& 4$ show interband transition associated between the valence and conduction electron state, an effect associated to the inner d orbital state with more energy than the conduction electron s state.

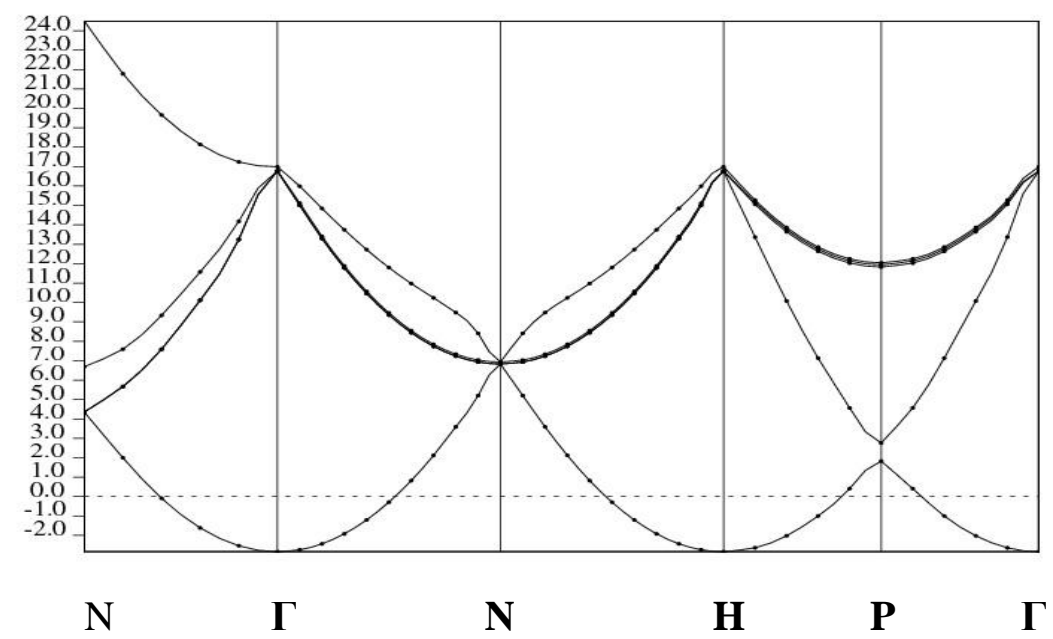

Figure 1: Electronic Structure of Sodium within DFT/LDA scheme. 


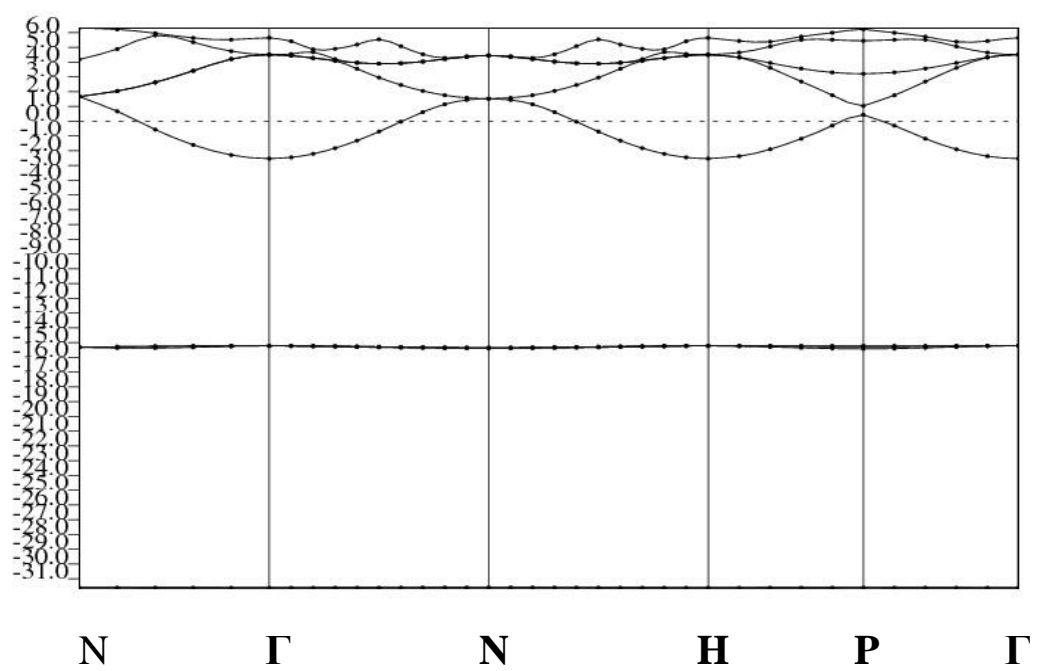

Figure 2: Electronic Structure of Potassium within DFT/LDA scheme.

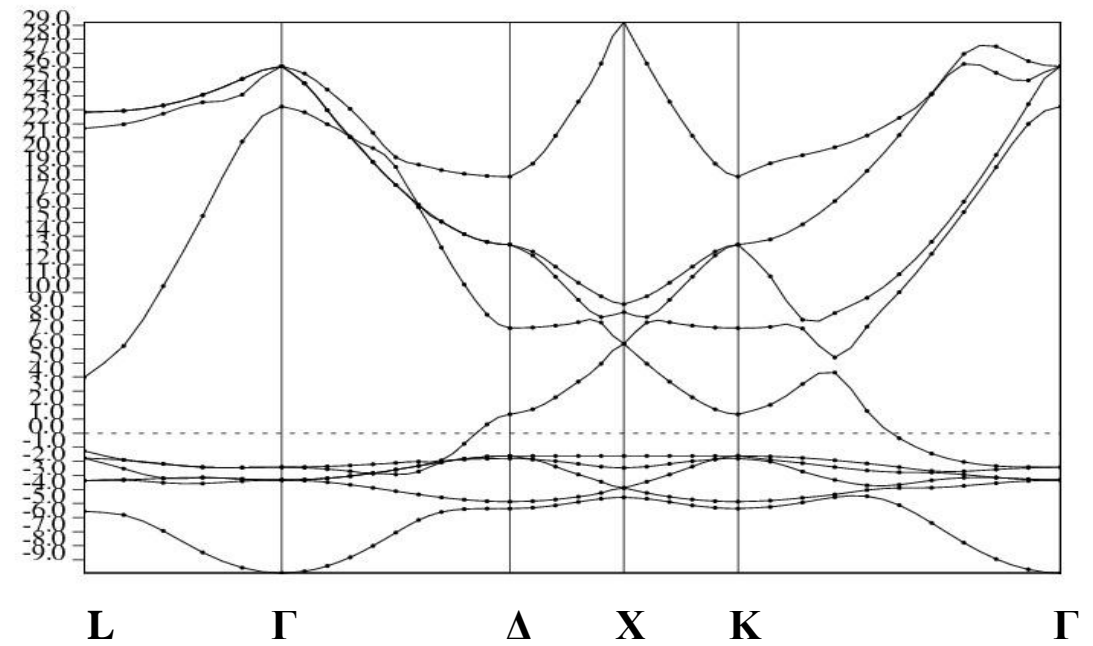

Figure 3: Electronic Structure of Copper within DFT/LDA scheme. 


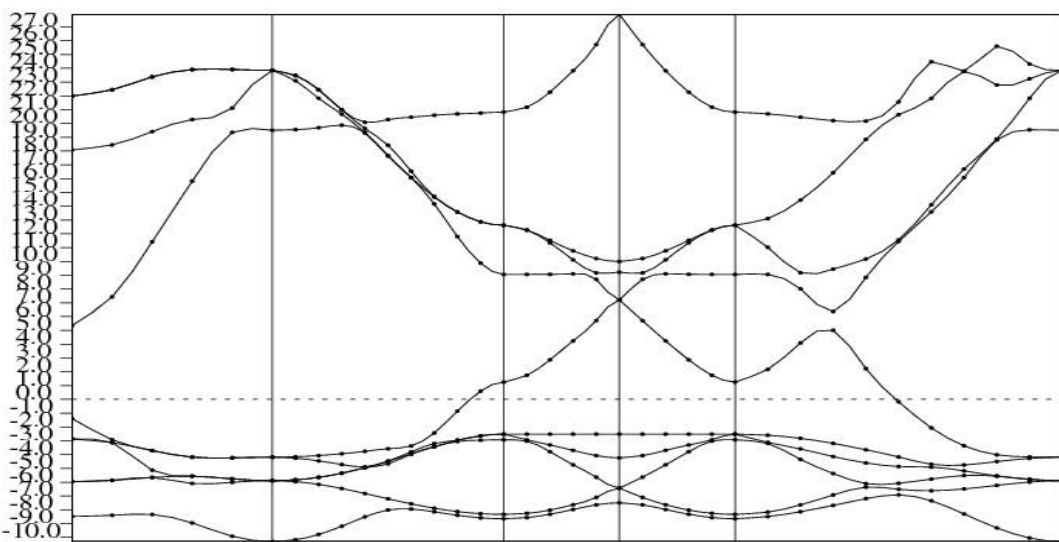

I. $\Gamma$

$\Lambda$

X

K

$\Gamma$

Figure 4: Electronic Structure of Copper within DFT/LDA scheme

\section{Conclusion}

Although the electronic structure shows the measure of excited electrons to the unoccupied states, density functional theory as a first principle method allow prediction of material properties and are often interpreted to correspond to electron energy levels. However the Fermi energy was observed inside several states, thereby allowing electrons that occupy states with slightly lower energies than the Fermi energy easily excited thermally or by electric field into states with energies in excess of the Fermi energy.

\section{References}

[1] Hohenberg, P., Kohn, W. Inhomogeneous Electron Gas, Phys. Rev. B 864 (1964) 136.

[2] Kohn, W., Sham, L. J. Self-Consistent Equations Including Exchange and Correlation Effects, Phys. Rev. A 1133 (1965) 140.

[3] Wing, K.L., Sukky, J., Q. Dong. Computational Nanomechanics of Materials, American Scientific Publishers, California, 2005.

[4] Barth, U. von. Basic Density-Functional Theory—an Overview, Physica Scripta 109 (2004) 9-39.

[5] Plummer, E.U., E. Jenseen. Experimental Band Structure of Na, Physical Review Letter 55 (1985) 1912.

[6] March, N., Lundqvist, S. Theory of inhomogenous Electron gas, Plenum, New York, 1983.

[7] Harrison, M. An Introduction to Density functional Theory, Imperial College, London, 2005.

[8] Perdew, J.P., Zunger, A. Self-interaction correction to density functional theory for many-electron systems, Phys. Rev. B 23 (1981).

[9] Monkhorst, H. J and Pack, J. D. Special points for Brillouin-zone integrations, Phys. Rev. B. 5188 (1976).

[10] Iannucci, P.A. A Brief Introduction to Band Structure in Three Dimensions, arXiv:1105.0220v1, 2011, 1-7. 